\title{
MICRODAMAGE ASSESSMENT OF BONE-CEMENT INTERFACES UNDER MONOTONIC AND CYCLIC COMPRESSION
}

\author{
Gianluca Tozzi, Qing-Hang Zhang, Jie Tong \\ Mechanical Behaviour of Materials Laboratory \\ School of Engineering \\ University of Portsmouth, UK
}

For correspondence:

Dr Gianluca Tozzi

Mechanical Behaviour of Materials Laboratory

School of Engineering

University of Portsmouth

Anglesea Building, Anglesea Road

Portsmouth PO1 3DJ

UK

Tel: +44-(0)23-9284-2514

Email: gianluca.tozzi@port.ac.uk 


\section{ABSTRACT}

Bone-cement interface has been investigated under selected loading conditions, utilising experimental techniques such as in situ mechanical testing and digital image correlation (DIC). However, the role of bone type in the overall load transfer and mechanical behaviour of the bone-cement construct is yet to be fully quantified. Moreover, microdamage accumulation at the interface and in the cement mantle has only been assessed on the exterior surfaces of the samples, where no volumetric information could be obtained. In this study, some typical bone-cement interfaces, representative of different fixation scenarios for both hip and knee replacements, were constructed using mainly trabecular bone, a mixture of trabecular and cortical bone and mainly cortical bone, and tested under static and cyclic compression. Axial displacement and strain fields were obtained by means of digital volume correlation (DVC) and microdamage due to static compression was assessed using DVC and FE, where yielded volumes and strains $\left(\varepsilon_{\mathrm{zz}}\right)$ were evaluated. A significantly higher load was transferred into the cement region when mainly cortical bone was used to interdigitate with the cement, compared with the other two cases. In the former, progressive damage accumulation under cyclic loading was observed within both the bone-cement interdigitated and the cement regions, as evidenced by the initiation of microcracks associated with high residual strains $\left(\varepsilon_{\text {zz_res }}\right)$.

Keywords: bone-cement interface, in situ mechanical testing, $\mu \mathrm{CT}$, microdamage, digital volume correlation, finite element analysis. 


\section{Introduction}

Bone-cement interface integrity has been identified as the critical element in the success and longevity of both hip and knee cemented replacements (Gardiner \& Hozack, 1994; Stocks et al., 1995; Thanner et al., 1999; Zant et al., 2008; Wang et al., 2009; Tozzi et al., 2012; Goodheart et al., 2014; Howard et al., 2014; Miller et al., 2014, 2014b). Principal concerns regarding the performance of cemented fixation in the hip were related to fatigue damage accumulation in the cement mantle in femoral (Cristofolini et al., 2007; Race et al., 2003, 2011) and acetabular (Heaton-Adegbile et al., 2006; Zant et al., 2007; Tong et al., 2008) components. To this extent it was shown (Cristofolini et al., 2007; Race et al., 2011) that fatigue damage accumulation within the cement mantle in femoral implants does not entirely depend on the mechanical competence of cement-implant interface, as previously thought (Jasty et al., 1991). In particular, it was observed (Race et al., 2003, 2011) that the majority of microcracks in the cement were related to the bone-cement interface, indicating how this interface can be directly compromised by local cement failures over time. However, to date there is only limited information on the early stages of crack formation and growth in the cement (Sinnett-Jones et al., 2009). On the acetabular side the cracking in the cement mantle seems to be limited compared to the femoral counterpart and other failure mechanisms, such as debonding at the bone-cement interface, are mainly responsible for the implant loosening (Tong et al., 2008, Zant et al., 2008; Wang et al., 2009; Tozzi et al., 2012). With regards to bone-cement fixation in the knee, a number of recent studies reported how the loss of interlock between bone and cement following in vivo service may eventually jeopardize the performance of both tibial (Goodheart et al., 2014; Miller et al., 2014, 2014b) and femoral (Howard et al., 2014) components over time. Goodheart et al. (2014) also showed how such ex vivo loss of interlock resulted in a reduced strength of the bone-cement fixation in total knee arthroplasty (TKA). In addition, it was observed that typical trabecular-cement constructs representative of TKA experienced localised failure within the supporting trabecular bone, under uniaxial compression. The latter observation is consistent with our previous work on the microdamage initiation and failure of bone-cement interface under monotonic compression, tension and shear, using specimens produced from trabecular bone and bone cement (Tozzi 
et al., 2012b, 2013, Zhang et al., 2014), where the failures were found mainly in the bone region, particularly at the bone-cement partially interdigitated region, rather than the fully interdigitated region. Moreover, under compressive loading, yielded bone volume was always found to be higher than that of the cement.

Extensive work has been conducted on bone-cement interface over the years, simulating different loading scenarios (Mann et al., 1997, 1999, 2001; Kim et al., 2004; Wang et al., 2010). Novel approaches, including digital image correlation (DIC) and in situ mechanical testing (Mann et al., 2008, 2009; Miller et al., 2010, 2011; Tozzi et al., 2012b, Goodheart et al., 2014) have been recently employed to explore local and micro-mechanisms. However, characterisation of the damage progression at the interface, particularly under cyclic loading conditions, has yet to be reported. Moreover, as most of the above mentioned studies focused on specific bone-cement constructs using either trabecular or cortical bones to interface with cement, the outcomes are often difficult to compare. In this context to study bone-cement composites built from different types of bones, representative of different cemented joint replacements (i.e. hip or knee), may be of interest.

Despite the overall reliability of traditional ex situ investigation techniques such as scanning electron microscopy (SEM) (Zant et al., 2007) or dye-penetrant for quantification of microdamage in the cement mantle (Cristofolini et al., 2007; Race et al., 2011), these methods are limited to the surface of the samples, where no 3D mapping of the crack surfaces could be interrogated (Race et al., 2003). With the recent and rapid progress of micro-focus computed tomography $(\mu \mathrm{CT})$ in conjunction with in situ mechanical testing (Nazarian \& Muller, 2004; Youssef et al., 2005; Buffiere et al., 2010), digital volume correlation (DVC) has become a powerful tool to examine full-field deformations in trabecular bone (Liu \& Morgan, 2007; Gillard et al., 2014; Dall'Ara et al., 2014), cortical bone (Dall'Ara et al., 2014), whole bones (Hussein et al., 2012, 2013) and cellular scaffolds (Madi et al., 2013). However, to date DVC has been mainly used in the analysis of solids and cellular materials, but little in known on the volumetric strain distribution in composite materials (Mortazavi et al., 2014) and there are no data with regards to bone-cement composites.

In this work, a comparative analysis of bone-cement interfaces built with different types of bone has been carried out using in situ mechanical testing, DVC and specimen-specific FE modelling. It is hoped that the work may provide useful 
information on the mechanical behaviour of different types of cemented joint replacements, such as largely cortical bones are generally found in femoral replacements, as opposed to trabecular bones in knee and acetabular replacements. The main aims of this paper are: 1) to investigate the microdamage evolution of three types of bone-cement interface, using mainly trabecular, a mixture of trabecular/cortical and mainly cortical bones under monotonic compression; and 2) to evaluate, for the stiffest cortical-cement type, the microcrack initiation in relation to the volumetric residual strain distribution in the interface and in the cement mantle under cyclic compression loading.

\section{Methods}

\subsection{Specimens}

Bovine bones were harvested from fresh iliac crest and used to interdigitate with acrylic bone cement (Simplex P, Stryker, UK), to create bone-cement interface samples. No selection was made with regards to trabecular or cortical structures and rectangular trabecular/cortical bone coupons were machined to size and thoroughly cleaned to remove fatty tissues. Bone-cement interface coupons were obtained using a method reported in Tozzi et al. (2012b). The bone cement preparation was conducted following the manufacturer's specifications. The cement dough was hand mixed with a plastic spatula for 60 s and then allowed to sit for 30 s to permit migration of bubbles to the surface for ablation (Macullay et al., 2002). The entire content was then applied onto a bone coupon placed into the lower half of a custom made mold and bone-cement interface strips were prepared by applying a constant pressure of $60 \mathrm{kPa}$ to the cement for approx $10 \mathrm{mins}$, following a procedure reported by Graham et al. (2003). Bone-cement samples were then cut out from the strip. Three bone-cement specimens with different trabecular/cortical composition named BC1 $(8 \mathrm{~mm} \times 6 \mathrm{~mm} \times 13.7 \mathrm{~mm}), \quad \mathrm{BC} 2 \quad(8 \mathrm{~mm} \times 6 \mathrm{~mm} \times 12.6 \mathrm{~mm})$ and BC3 $(8 \mathrm{~mm} \times 6 \mathrm{~mm} \times 11.7 \mathrm{~mm})$ were selected to represent a typical range of interdigitated bone structures in joint fixation (Fig. 1). 


\subsection{Experimental procedures}

In situ compression testing of bone-cement samples $(\mathrm{n}=3)$ in combination with time-lapsed $\mu \mathrm{CT}$ imaging was performed. The specimens were firstly imaged using $\mu C T$ (CT X-Ray Inspection System, X-Tek Systems Ltd). The $\mu$ CT scanner was set to a voltage of $55-80 \mathrm{kV}$ and a current of $110-150 \mu \mathrm{A}$. With an isotropic voxel size of $20 \mu \mathrm{m}$ the image acquisition was performed at a rotational step of $0.19^{\circ}$ over $360^{\circ}$ for 90 min approximately. The 3D reconstruction of the samples was obtained using VG StudioMax 2.0 (Volume Grapics, GmbH). The bone volume fraction (BV/TV), which is a measure of the bone tissue density within the sample, was calculated by dividing the total bone volume within the volume of interest containing only bone material, over the volume of interest itself. The values of BV/TV for BC1, BC2 and BC3 were obtained.

All specimens were monotonically compressed up to a global strain of $4 \%$, following a small preload of $10 \mathrm{~N}$. The corresponding displacements achieved for $\mathrm{BC} 1, \mathrm{BC} 2$ and $\mathrm{BC} 3$ were approximately $0.54 \mathrm{~mm}, 0.5 \mathrm{~mm}$ and $0.46 \mathrm{~mm}$, respectively. Whilst under displacement, the specimen was allowed to relax to a steady-state for approx 15mins before imaging and data acquisition was carried out. All tests were conducted under displacement control at a constant cross-head speed of $0.01 \mathrm{~mm} / \mathrm{s}$.

A maximum strain of $4 \%$ was used for the cyclic test at a load ratio of zero. This level of strain corresponds approximately to $73 \%$ of the compressive strength of the same type of samples, previously tested in our lab under monotonic loads. The bottom platen used in the loading stage with $\mathrm{BC} 3$ in place was mounted on a servohydraulic Si-Plan $(5 \mathrm{kN})$ and loaded to uniaxial cyclic compression against an exact copy of the top platen used in the stage. This procedure was followed in order to minimize the misalignment during sequential $\mu C T$ examinations. The same small preload of $10 \mathrm{~N}$ was applied to ensure full contact with the platens and the subsequent test was carried out, using a sinusoidal loading waveform, under the same global strain (4\%) at a frequency of $5 \mathrm{~Hz}$ (Kim et al., 2004). The test was stopped to allow $\mu \mathrm{CT}$ scanning of the sample (preloaded only) at selected intervals of 10,100 and 200 cycles, respectively. 
Both 'end' and 'side' artefacts were minimised by examining an internal core volume of the original reconstructions, corresponding to a reduction of approximately $10 \%$ for each dimension of the sample (Fig.1).

\subsection{Digital volume correlation (DVC)}

LaVision DVC software (Davis, 8.0.1, Germany), with an implemented quasilocal predictor-corrector correlation algorithm based on fast Fourier transform (FFT) cross-correlation to compare sub-volumes, was used to compute the $3 \mathrm{D}$ displacement $\left(v_{z}\right)$ and strain fields for both static $\left(\varepsilon_{z z}\right)$ and cyclic residual $\left(\varepsilon_{z z}\right.$ res $)$ components.

For the static loading cases, a pair of images for all samples were taken at the preload and the deformed stages, namely: the reference reconstructed image obtained in the preload stage, and the deformed image obtained after compressing the specimen to a global $4 \%$ strain. For the cyclic cases, the preloaded images of BC3 following the selected cycling steps (1cyc, 10cyc, 100cyc, 200cyc) were used in order to compute the accumulated residual strains ( $\left.\varepsilon_{\text {zz_res }}\right)$ along the $Z$ direction.

DVC computation produced final sub-volumes of $32 \times 32 \times 32$ voxels $^{3}$ overlapped by $50 \%$, reached after successive (predictor) passes using sub-volumes of $96 \times 96 \times 96$ voxels $^{3}$ and $64 \times 64 \times 64$ voxels $^{3}$. From the displacements at the centre of the sub-volumes, all the components of the Green-Lagrange strain tensor were calculated using a centered finite differences scheme (Germaneau et al., 2008).

For visualisation, the displacement $\left(\mathrm{v}_{\mathrm{z}}\right)$ and strain vector fields $\left(\varepsilon_{\mathrm{zz}}, \varepsilon_{\mathrm{zz}}\right.$ res $)$ were imported into AVIZO 6.3 (VSG, France).

\subsection{Finite element modelling}

For each of the three bone-cement composite specimens, $\mu \mathrm{CT}$ scan images (Fig.1) were imported into AVIZO 6.3 (VSG, France) for three-dimensional reconstruction and FE mesh generation and detailed procedures have been reported elsewhere (Zhang et al. 2014). In brief, the bone and the cement bodies were constructed and meshed with four-node tetrahedral elements separately, where the cement volume was shrunk by one voxel $(20 \mu \mathrm{m})$ before meshing to ensure a good apposition of the two bodies. The meshes of the two bodies were then imported into 
ABAQUS 6.12 (Dassault Systemes, USA) to form the bone-cement composite assembly for nonlinear analysis. The three bone-cement composite models: $\mathrm{BC} 1$, BC2 and BC3, consist of 1,291,437 elements (342,019 nodes), 1,333,484 elements (328,816 nodes) and 1,569,986 elements (379,073 nodes), respectively (Fig.2).

The cortical and trabecular bone tissues were modelled as elastic-plastic materials, with an asymmetric yield strain of $0.6 \%$ in tension and $1 \%$ in compression (Niebur et al., 2000). The elastic moduli of the cortical bone and trabecular bone were assumed as 9,000MPa and 5,000MPa, respectively, while the Poisson's ratio was assumed to be 0.3 (Szabó et al., 2011). A similar asymmetrical elastic-plastic constitutive law was also used for the cement material, of which the elastic modulus, Poisson's ratio, yield stress under tension and yield stress under compression were assumed to be 3,000MPa, 0.33, 30MPa, and 70MPa, respectively (Kuehn et al., 2005; Kurtz et al., 2005). The interaction between the contact surface of the bone and that of the cement was modelled as surface-to-surface finite sliding contact, with a friction coefficient of 0.4 (Zhang et al., 2014).

FE analyses were performed using large deformation to account for the geometrical nonlinearity and boundary conditions to mimic those in the experiments. The bottom surface of the cement was fully constrained in all degrees of freedom while a uniaxial static displacement in compression was applied incrementally to the top surface of bone up to $0.2 \mathrm{~mm}$. The predicted apparent behaviour and local damage evolution were obtained to compare with the experimental results.

\section{Results}

The bone volume fraction (BV/TV) calculated for the three samples, BC1, BC2 and $\mathrm{BC} 3$, are $0.14,0.62$ and 0.75 , respectively. The mechanical performance under static compression (Fig.3a) seems to be directly related to the BV/TV, which enhances with the increase in the amount of cortical bone in the composite. The predicted stress-displacement responses for the three specimens are comparable with their subject-specific experimental curves, without the initial toe region (Fig.3b). Consistent with experimental observation, the effect of bone type is significant on the bone-cement composite response under compression. The predicted elastic stiffness 
of $\mathrm{BC} 3$ is 1.9 and 11.0 times higher than that of $\mathrm{BC} 2$ and $\mathrm{BC} 1$, respectively, while the corresponding ratios from experiments are 1.6 and 9.9.

The volumetric displacement $\left(\mathrm{v}_{\mathrm{z}}\right)$, strain maps $\left(\varepsilon_{\mathrm{zz}}\right)$ calculated from the DVC and the predicted strains $\left(\varepsilon_{z z}\right)$ from the FE for the three bone-cement interfaces also confirmed that an increased BV/TV, due to the increased amount of cortical bone, resulted in a significantly higher load transfer across the interface (Fig.4a, b, c). In particular, the highest compressive strains $\left(\varepsilon_{\mathrm{zz}}\right)$ are found to be associated with localised damage occurred in trabecular bone for both BC1 and BC2 (Fig.5a, b). On the other hand, BC3 experienced lower strains $\left(\varepsilon_{\mathrm{zz}}\right)$ which are mainly distributed in the interdigitated and cement regions (Fig.5c).

Fig. 6 shows the predicted distribution of yielded elements in the three models under a compressive displacement of $0.2 \mathrm{~mm}$. It is obvious that although the yielded bone volumes are higher than those of cement, the locations of high strain concentration and yielded cement volumes differ significantly among the three models. The predominant deformation for $\mathrm{BC} 1$ occurred in the bone region, whereas the cement region appeared to be unaffected when the pure trabecular bone was interdigitated with cement. In $\mathrm{BC} 2$, yielding of the bone mainly occurred in the interdigitated region and much more load was transferred to the cement body. Cement yielding was observed in BC3 where cortical bone dominated the bonecement composite. As shown in Fig.7, the predicted percentages of yielded cement volume within the interdigitated region (Fig.7a) are $0.04 \%, 2.60 \%$ and $17.12 \%$ for $\mathrm{BC} 1, \mathrm{BC} 2$ and $\mathrm{BC} 3$, respectively. Within the bulk cement region (Fig.7b), the corresponding values are $0 \%, 0.23 \%$ and $4.75 \%$, respectively.

Under cyclic compression, the overall stiffness of BC3 was virtually unaffected up to 100 cycles from the experiment. From this point onwards a gradual reduction of stiffness was observed, resulting in a decrease of $23 \%$ at 200 cycles compared to the original stiffness value (Fig.8). The volumetric strain map showed in Fig.9 confirms the presence of relatively high levels of residual strain ( $\left.\varepsilon_{\mathrm{zz} \_ \text {res }}\right)$ accumulated in both interdigitated and cement regions of the sample after 200 cycles. Furthermore, the regions with high residual strains $\left(\varepsilon_{\text {zz_res }}\right)$ correspond to the evident microdamage in the cement (Fig.10a), mainly due to the presence of pre-existing voids. The bone and bone-cement interdigitated regions also show microdamage 
associated with compressive residual strains $\left(\varepsilon_{z_{z} \text { res }}\right)$, which seems to favour separation at the interface (Fig.10b).

\section{Discussion}

The first aim of this study is to investigate the microdamage evolution under monotonic compression for three types of bone-cement interfaces, generated using different proportions of trabecular/cortical bones representative of a range of bone stocks for cemented fixation in femoral hip and knee. The overall behaviour of the samples shown in Fig.3 may be explained by the volumetric displacement $\left(v_{z}\right)$ and strain ( $\left.\varepsilon_{\mathrm{zz}}\right)$ maps (Fig.4), where the load supported by the bone-cement composite in $\mathrm{BC} 1$ seems to be much lower due to the predominant presence of trabecular bone type with low BV/TV. Similar findings were recently reported by Goodheart et al. (2014) for bone-cement constructs from the tibial tray of knee replacements. It was found that under uniaxial compressive loading, freshly-cemented specimens had low strains in the cement and at the bone-cement interface compared to the supporting trabecular bone. Conversely, the relatively high BV/TV in samples BC2 and BC3 resulted in increased compressive stiffness and strength, consistent with the previous findings from Janssen et al. (2009).

Similarly to our previous studies (Tozzi et al., 2012b, 2013), microdamage in the BC1 and BC2 composites (Fig.5) is mainly characterised by bending and buckling of trabeculae in different locations of the bone region. The local DVC computation (Fig.5a, b) allowed a successful coupling of a qualitative microdamage inspection, to a quantitative information from the compressive strain fields $\left(\varepsilon_{z z}\right)$, throughout the entire volume of the bone-cement samples. The microdamage in the cement region of $\mathrm{BC} 3$ under static compression (Fig.5c) was more difficult to appreciate visually and produced low strain distribution $\left(\varepsilon_{z z}\right)$.

Consistent with the DVC observation, the results obtained from the FE simulation show that the higher strength of the bone is generally related to a higher risk of failure in the bone-cement interdigitated region. For $\mathrm{BC} 1$, the calculated percentages of yielded bone and cement volumes in the interdigitated region under a $0.2 \mathrm{~mm}$ compressive displacement are $6.9 \%$ and $0.04 \%$, respectively, while the corresponding values are increased significantly to $78.6 \%$ and $17.1 \%$ in $\mathrm{BC} 3$. This 
may indicate that the load transferred between the bone and the cement was more efficient in $\mathrm{BC} 3$ than in $\mathrm{BC} 1$, resulting in more deformed interdigitated and cement regions in $\mathrm{BC} 3$. The failure of bone and cement in $\mathrm{BC} 1$ is consistent with the findings of our previous studies (Tozzi et al., 2012b; Zhang et al., 2014), where the damaged bone volumes are always higher than those of cement under compression, despite the different bone morphologies interdigitated with the cement. On the other hand, the bone-cement responses observed under tension and shear (Waanders et al., 2010; Zhang et al., 2014) suggest that damaged bone volumes are higher than cement only when low density trabecular bone is used to form the interface.

The second aim is to assess the microdamage initiation at the bone-cement interface and in the cement mantle for cortical-cement type (BC3) under cyclic compression. Unlike previous fatigue studies on bone-cement interfaces (Mann et al., 2009; Waanders et al., 2009, 2010b) under shear or tension, a relatively high stress was applied to the specimen and the responses were monitored for a limited number of cycles. The peak compressive stress of about 50MPa in BC3 (Fig.8) represents $73 \%$ of monotonic compressive strength, significantly higher than $40-50 \%$ of monotonic strength normally used for fatigue testing. This was adopted simply to accelerate the fatigue experiments, so that the test duration would be more manageable. Regarding the choice of 200 cycles, this was mainly dictated by the first evidence of microdamage accumulated in the structure. Bone tissue and cement may experience plastic deformation under compression (Kurtz et al., 2005; Lewis, 1997). Thus microdamage accumulation represented by residual stress, rather than brittle cracking, may be more appropriate for such loading conditions. Cyclic compressive loading on BC3 resulted in a reduced overall stiffness after 200 cycles (Fig.8), which is consistent with the residual strain $\left(\varepsilon_{\text {zz_res }}\right)$ obtained by DVC (Fig.9d). From this perspective, the load transfer observed in the static case for BC3 (Fig.4c) resulted in microdamage accumulation, which was mainly localised in the cement (Fig.10a) and in the bone-cement interdigitated (Fig.10.b) regions. These findings suggest how the presence of cortical bone at the interface would not only favor bone-cement separation, but also promote cyclic damage accumulation in the cement mantle, as a result of microcracks formation and progression in accordance with previous observations (Cristofolini et al., 2007; Mann et al., 2008; Race et al., 2003, 2011). Moreover, the current results seem to support the assumption in which 
voids and pores in the cement mantle act as crack initiators for the cyclic damage accumulation process (Murphy \& Prendergast, 2002; Race et al., 2011; SinnettJones et al., 2009), although there is no clear consensus on the role of these in the eventual contribution to macrofracture in the cement (Janssen et al., 2005; HeatonAdegblile et al., 2006).

There are limitations in the current work. Firstly, only a small number of samples were tested under static and cyclic compressive loadings, thus it is not possible to present any statistical evaluations, but only a general trend of microdamage evolution in bone-cement interfaces built with selected types of bones. Secondly, DVC calculation is subjected to displacement and strain uncertainties that may vary in relation to different factors such as noise of the $\mu \mathrm{CT}$ system, imaging parameters, sample structure and sub-volume size (Dall'Ara et al., 2014; Gillard et al., 2014; Roberts et al., 2014). This analysis was not carried out in the current study and DVC performance evaluation is therefore needed to better quantify displacement and strain fields, produced in different bone-cement constructs.

\section{Conclusions}

The overall behaviour at the bone-cement interface seems to be dependent on the type of the bone, represented by BV/TV, used to interdigitate with the cement. The microdamage evolution at the interface under monotonic compression has been well captured by both DVC and FE analysis. In particular, higher yielded volumes and strains $\left(\varepsilon_{z z}\right)$ are predominantly localised in the trabecular bone structure, resulting in both bending and buckling at trabeculae level. The presence of a stiffer cortical bone produced more effective load transfer through the interface into the cement region, thus, resulting in a damage accumulation within both bone-cement interdigitated and cement regions under cyclic compression. In this case, volumetric residual strain obtained from DVC seems to be indicative of microdamage initiation in the interface and in the cement mantle. The results reported in this study may provide useful information on the type of failures that would be likely to occur for different bone-cement interfaces, in femoral hip and knee replacements, for example. 


\section{Conflict of interest statement}

None.

\section{Acknowledgements}

The authors gratefully acknowledge Stryker UK, for donating the bone cement. Numerical computations were performed on the Sciama High Performance Compute (HPC) cluster, which is supported by the ICG, SEPNet and the University of Portsmouth.

\section{REFERENCES}

Buffière, J.Y., Maire, E., Adrien, J., Masse, J.P., Boller, E., 2010. In Situ Experiments with $\mathrm{X}$ ray Tomography: an Attractive Tool for Experimental Mechanics. Exp. Mech. 50(3), 289-95.

Cristofolini, L., Erani, P., Savigni, P., Bordini, B., Viceconti, M., 2007. Preclinical assessment of the long-term endurance of cemented hip stems. Part 2: in-vitro and ex-vivo fatigue damage of the cement mantle. Proceedings of the Institution of Mechanical Engineers, Part H: Journal of Engineering in Medicine. 221: 585.

Dall'Ara, E., Barber, D., Viceconti, M., 2014. About the inevitable compromise between spatial resolution and accuracy of strain measurement for bone tissue: A 3D zero-strain study. J. Biomech. in press.

Gardiner, R.C., Hozack, W.J., 1994. Failure of the cement-bone interface. A consequence of strengthening the cement-prosthesis interface? J. Bone Joint Surg. Br. 76(1), 49-52.

Germaneau, A., Doumalin, P., Dupré, J.-C., 2008. Comparison between X-ray microcomputed tomography and optical scanning tomography for full 3D strain measurement by digital volume correlation. NDT\&E International. 41(6), 407-15.

Gillard, F., Boardman, R., Mavrogordato, M., Hollis, D., Sinclair, I., Pierron, F., Browne, M., 2014. The application of digital volume correlation (DVC) to study the microstructural behaviour of trabecular bone during compression. Journal of the Mechanical Behavior of Biomedical Materials. 29, 480-99. 
Goodheart, J.R., Miller, M.A., Mann, K.A., 2014. In Vivo Loss of Cement-Bone Interlock Reduces Fixation Strength in Total Knee Arthroplasties. J. Orthop. Res. 32, 1052-60.

Graham, J., Ries, M., Pruitt, L., 2003. Effect of bone porosity on the mechanical integrity of the bone-cement interface. J. Bone and Joint Surg. 85(10), 1901-8.

Heaton-Adegbile, P., Zant, N.P., Tong, J., 2006. In vitro fatigue behaviour of a cemented acetabular reconstruction. J. Biomech. 39, 2882-86.

Howard, K.I., Miller, M.A., Damron, T.A., Mann, K.A., 2014. The Distribution of Implant Fixation for Femoral Components of TKA: A Postmortem Retrieval Study. J. Arthroplasty. in press.

Hussein, A.I., Barbone, P.E., Morgan, E.F., 2012. Digital volume correlation for study of the mechanics of whole bones. Procedia IUTAM: Full Field Measurements and Identication in Solid Mechanics. 4, 116-25.

Hussein, A.I., Mason, Z.D., Morgan, E.F., 2013. Presence of intervertebral discs alters observed stiffness and failure mechanisms in the vertebra. J. Biomech. 46, 1683-88.

Janssen, D., Aquarius, R., Stolk, J., Verdonschot, N., 2005. The contradictory effects of pores on fatigue cracking of bone cement. J. Biomed. Mater. Res. B Appl. Biomater. 74, 747-53.

Janssen, D., Mann, K.A., Verdonschot, N., 2009. Finite element simulation of cement-bone interface micromechanics: a comparison to experimental results. J Orthop. Res. 27(10), 1312-8.

Jasty, M., Maloney, W.J., Bragdon, C.R., O'Connor, D.O., Haire, T., Harris, W.H., 1991. The initiation of failure in cemented femoral components of hip arthroplasties. Journal of Bone \& Joint Surgery - British. 73, 551-8.

Kim, D.G., Miller, M.A., Mann, K.A., 2004. Creep dominates tensile fatigue damage of the cement-bone interface. J. Orthop. Res. 22, 633-640.

Kuehn, K.D., Ege, W., Gopp, U., 2005. Acrylic bone cements: mechanical and physical properties. The Orthopedic Clinics of North America. 36, 29-39. 
Kurtz, S.M., Villarraga, M.L., Zhao, K., Edidin, A.A., 2005. Static and fatigue mechanical behavior of bone cement with elevated barium sulfate content for treatment of vertebral compression fractures. Biomaterials. 26, 3699-3712.

Lewis, G., 1997. Properties of acrylic bone cement: State of the art review. Journal of Biomedical Materials Research. 38(2), 155-182.

Liu, L., Morgan, E.F., 2007. Accuracy and precision of digital volume correlation in quantifying displacements and strains in trabecular bone. J. Biomech. 40(15), 351620.

Macaulay, W., DiGiovanni, C.W., Restrepo, A., Saleh, K.J., Walsh, H., Crossett, L.S., Peterson, M.G., Li, S., Salvati, E.A., 2002. Differences in bone-cement porosity by vacuum mixing, centrifugation, and hand mixing. J. Arthroplasty. 17, 569-75.

Madi, K., Tozzi, G., Zhang, Q.-H., Tong, J., Cossey, A., Au, A., Hollis, D., Hild, F., 2013. Computation of full-field displacement in a scaffold implant using Digital Volume Correlation and Finite Element Analysis. Med. Eng. \& Phys. 35(9), 12981312.

Mann, K.A., Ayers, D.C., Werner, F.W., Nicoletta, R.J., Fortino, M.D., 1997. Tensile strength of the cement-bone interface depends on the amount of bone interdigitated with PMMA cement. J. Biomech. 30, 339-346.

Mann, K.A., Werner, F.W., Ayers, D.C., 1999. Mechanical strength of the cementbone interface is greater in shear than in tension. J. Biomech. 32, 1251-1254.

Mann, K.A., Mocarski, R., Damron, L.A., Allen, M.J., Ayers, D.C., 2001. Mixed-mode failure response of the cement-bone interface. J. Orthop. Res. 19, 1153-1161.

Mann, K.A., Miller, M.A., Clearly, R., Janssen, D., Verdonschot, N., 2008. Experimental micromechanics of the cement-bone interface. J. Orthop. Res. 26(6), 872-79.

Mann, K.A., Miller, M.A., Race, A., Verdonschot, N., 2009. Shear fatigue micromechanics of the cement-bone interface: an in vitro study using digital image correlation techniques. J. Orthop. Res. 27(3), 340-6. 
Miller, M.A., Eberhardt, A.W., Clearly, R., Verdonschot, N., Mann, K.A., 2010. Micromechanics of postmortem-retrieved cement-bone interfaces. J. Orthop. Res. 28(2), 170-7.

Miller, M.A., Race, A., Waanders, D., Clearly, R., Janssen, D., Verdonschot, N., Mann, K.A., 2011. Multi-axial loading micromechanics of the cement-bone interface in post-mortem retrievals and lab-prepared specimens. J. Mech. Behav. Biomed. Mater. 4, 366-74.

Miller, M.A., Terbush, M.J., Goodheart, J.R., Izant, T.H., Mann, K.A., 2014. Increased initial cement-bone interlock correlates with reduced total knee arthroplasty micro-motion following in vivo service. J. Biomech. 47, 2460-66.

Miller, M.A., Goodheart, J.R., Izant, T.H., Rimnac, C.M., Cleary, R.J., Mann, K.A., 2014b. Loss of Cement-bone Interlock in Retrieved Tibial Components from Total Knee Arthroplasties. Clin. Orthop. Relat. Res. 472, 304-13.

Mortazavi, F., Ghossein, E., Lévesque, M., Villemure, I., 2014. High resolution measurement of internal full-field displacements and strains using global spectral digital volume correlation. Optics and Lasers in Engineering. 55, 44-52.

Murphy, B.P., Prendergast, P.J., 2002. The relationship between stress, porosity and nonlinear damage accumulation in acrylic bone cement. J. Biomed. Mater. Res. 59, 646-54.

Nazarian, A., Muller, R., 2004. Time-lapsed microstructural imaging of bone failure behavior. J. Biomech. 37, 55-65.

Niebur, G.L., Feldstein, M.J., Yuen, J.C., Chen, T.J., Keaveny, T.M., 2000. Highresolution finite element models with tissue strength asymmetry accurately predict failure of trabecular bone. Journal of Biomechanics. 33, 1575-1583.

Race, A., Miller, M.A., Ayers, D.C., Mann, K.A., 2003. Early cement damage around a femoral stem is concentrated at the cement/bone interface. J. Biomech. 36, 48996.

Race, A., Miller, M.A., Izant, T.H., Mann, K.A., 2011. Direct evidence of 'damage accumulation' in cement mantles surrounding femoral hip stems retrieved at autopsy: Cement damage correlates with duration of use and BMI. J. Biomech. 44, 2345-50. 
Roberts, B.C., Perilli, E., Reynolds, K.J., 2014. Application of the digital volume correlation technique for the measurement of displacement and strain fields in bone: A literature review. J. Biomech. 47, 923-34.

Sinnett-Jones, P.E., Browne, M., Moffat, A.J., Jeffers, J.R.T., Saffari, N., Buffiere, J.Y., Sinclair, I., 2009. Crack initiation processes in acrylic bone cement. J. Biomed. Mater. Res. A. 89, 1088-97.

Stocks, G.W., Freeman, M.A., Evans, S.J. 1995. Acetabular cup migration: prediction of aseptic loosening. J. Bone Joint Surg. Br. 77, 853-861.

Stolk, J., Verdonschot, N., Cristofolini, L., Toni, A., Huiskes, R., 2002. Finite element and experimental models of cemented hip joint reconstructions can produce similar bone and cement strains in pre-clinical tests. J. Biomech. 35, 499-510.

Stolk, J., Verdonschot, N., Mann, K.A., Huiskes, R., 2003. Prevention of meshdependent damage growth in finite element simulations of crack formation in acrylic bone cement. J. Biomech. 36, 861-871.

Szabó, M.E., Zekonyte, J., Katsamenis, O.L., Taylor, M., Thurner, P.J., 2011. Similar damage initiation but different failure behavior in trabecular and cortical bone tissue. Journal of The Mechanical Behavior of Biomedical Materials. 4, 1787-1796.

Thanner, J., Karrholm, J., Malchau, H., Herberts, P., 1999. Poor outcome of the PCA and Harris-Galante hip prostheses. Randomized study of 171 arthroplasties with 9year follow-up. Acta Orthop. Scand. 70, 155-162.

Tong, J., Zant, N.P., Wang, J.-Y., Heaton-Adegbile, P., Hussell, J.G., 2008. Fatigue in cemented acetabular replacements. Int. J. Fatigue. 30(8), 1366-1375.

Tozzi, G., Lupton, C., Heaton-Adegbile, P., Tong, J., 2012. Damage evolution in acetabular reconstructs under physiological testing in a saline environment. J. Biomech. 45, 405-408.

Tozzi, G., Zhang, Q.-H., Tong, J., 2012b. 3D real-time micromechanical compressive behaviour of bone-cement interface: experimental and finite element studies. J. Biomech. 45, 356-363. 
Tozzi, G., Zhang, Q.-H., Tong, J., Guillen, T., Ohrndorf, A., Christ, H.-J., 2013. Mechanical characterisation of a metallic foam-cement composite under selected loading conditions. J. Mater. Sci. Mater. Med. 24(11), 2509-2518.

Waanders, D., Janssen, D., Miller, M.A., Mann, K.A., Verdonschot, N., 2009. Fatigue creep damage at the cement-bone interface: an experimental and a micromechanical finite element study. Journal of Biomechanics, 42, 2513-9.

Waanders, D., Janssen, D., Mann, K.A., Verdonschot, N., 2010. The mechanical effects of different levels of cement penetration at the cement-bone interface. J Biomech. 43(6):1167-75.

Waanders, D., Janssen, D., Mann, K.A., Verdonschot, N., 2010b. The effect of cement creep and cement fatigue damage on the micromechanics of the cementbone interface. J Biomech. 43, 3028-34.

Wang, J.-Y., Heaton-Adegbile, P., New, A., Hussell, J.G., Tong, J., 2009. Damage evolution in acetabular replacements under long-term physiological loading conditions. Journal of Biomechanics, 42, 1061-68.

Wang, J.Y., Tozzi, G., Chen, J., Contal, F., Lupton, C., Tong, J., 2010. Bone-cement interfacial behaviour under mixed mode loading conditions. J. Mech. Behav. Biomed. Mater. 3, 392-398.

Youssef, S., Maire, E., Gaertner, R., 2005. Finite element modeling of the actual structure of cellular materials determined by X-ray tomography. Acta Mat. 53(3), 719-30.

Zhang, Q.-H., Tozzi, G., Tong, J., 2014. Micromechanical damage of trabecular bone-cement interface under selected loading conditions: a finite element study. Computer Methods in Biomechanics and Biomedical Engineering. 17(3), 230-8.

Zant, N.P., Wong, C.K.Y, Tong, J., 2007. Fatigue failure in the cement mantle of a simplified acetabular replacement model. Int. J. Fatigue. 29, 1245-52.

Zant, N.P., Heaton-Adegbile, P., Hussell, J.G., Tong, J., 2008. In vitro fatigue failure of cemented acetabular replacements: a hip simulator study. J. Biomech. Eng. Trans. ASME 130, 021019-1-9. 
Figure captions:

Fig.13D reconstruction of the three bone-cement specimens generated from $\mu \mathrm{CT}$ images.

Fig.2 The finite element mesh of the bone-cement models with details of the interdigitated regions (-inter) of the three specimens (a) BC1; (b) BC2 and (c) BC3. The bone and cement are represented with red and blue colour, respectively.

Fig.3 (a) The original experimental stress-displacement curves of the three specimens; (b) a comparison between the modified experimental curves (without the toe region) and the corresponding FE predictions.

Fig.4 The 3D visualisation of displacement vz (top), strain $\varepsilon z z$ (middle) maps from DVC under a $4 \%$ global strain ( $Z$ direction) and predicted $\varepsilon Z z$ distribution from FE under $0.2 \mathrm{~mm}$ displacement (bottom) for the three specimens (a) BC1, (b) BC2 and (c) BC3.

Fig.5 Strain maps (left) and 3D reconstruction of selected sub-volumes from the three specimens in the preloaded state (middle) and at $4 \%$ strain (right): (a) BC1; (b) $\mathrm{BC} 2$ and (c) $\mathrm{BC} 3$, where microdamage appear to be associated with regions of high vertical strain (عzz). Arrows indicate the locations of some of the microdamages.

Fig.6 The predicted distribution of yielded elements at a displacement of $0.2 \mathrm{~mm}$ for the three bone-cement models (a) BC1; (b) BC2 and (c) BC3. The bone and cement are represented with red and blue colour, respectively.

Fig.7 The predicted percentages of yielded volumes accumulated in the cement within the interdigitated region (a) and bulk cement region (b), as a function of the applied displacement. The percentage value was obtained by dividing the volume of the yielded cement volume by the corresponding whole cement volume in the same region.

Fig. 8 The evolution of the stress-displacement curve of BC3 under a global strain of $4 \%$ at selected loading cycles. The maximum stress reached during the first cycle is approx $73 \%$ of the failure stress under a monotonic loading for this type of sample. 
Fig.9 The 3D visualization of the accumulated residual strain (Eres_zz) from the DVC analysis of BC3 at (a) cycle 1; (b) cycle 10; (c) cycle 100 and (d) cycle 200. The nominal cyclic strain range is $4 \%, R=0$.

Fig.10 The residual strain maps (left) and the 3D reconstructions of selected subvolumes from the side (a) and the front (b) part of $\mathrm{BC} 3$ when preloaded at 0 cycles (middle) and after 200 cycles (right), where microdamages were identified with regions of high residual strain (Eres_zz). Arrows indicate some microdamages. The nominal cyclic strain range is $4 \%, R=0$.

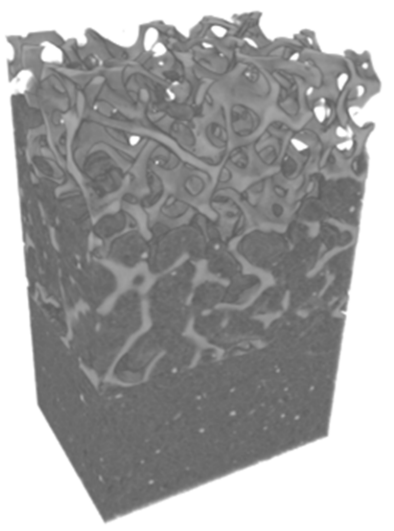

$\mathrm{BC} 1$

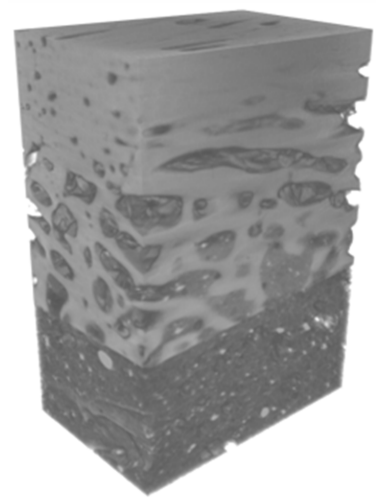

$\mathrm{BC} 2$

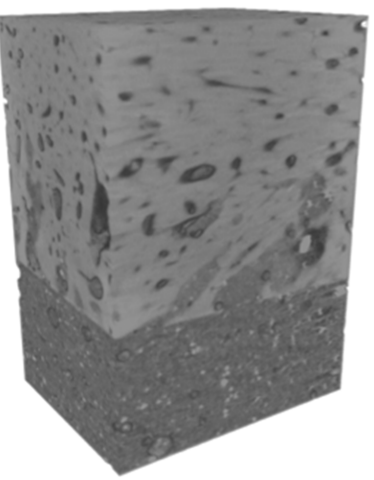

$\mathrm{BC} 3$

Fig.1 
(a)

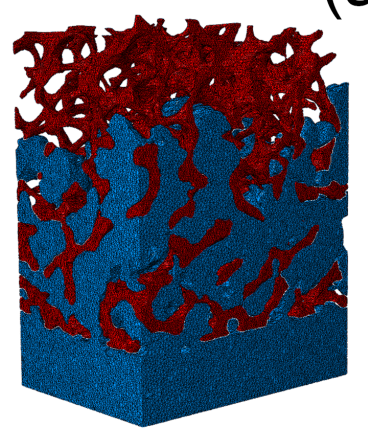

BC1

(b)

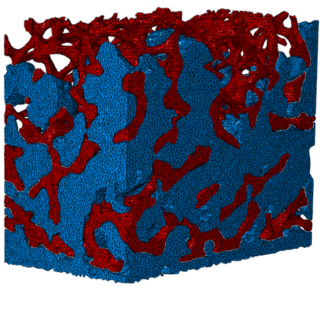

BC1-inter

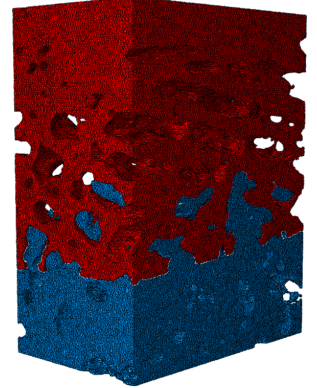

$\mathrm{BC} 2$

(C)

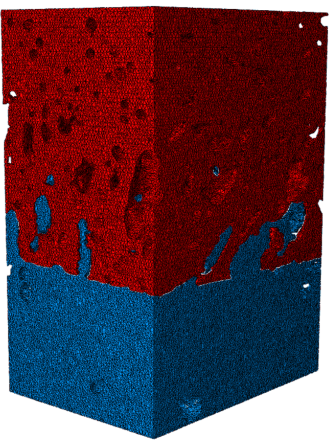

BC3

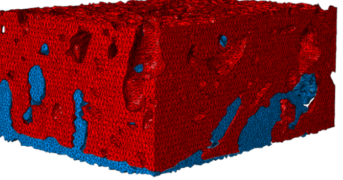

BC3-inter

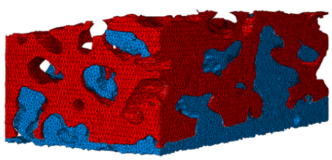

BC2-inter

Fig. 2

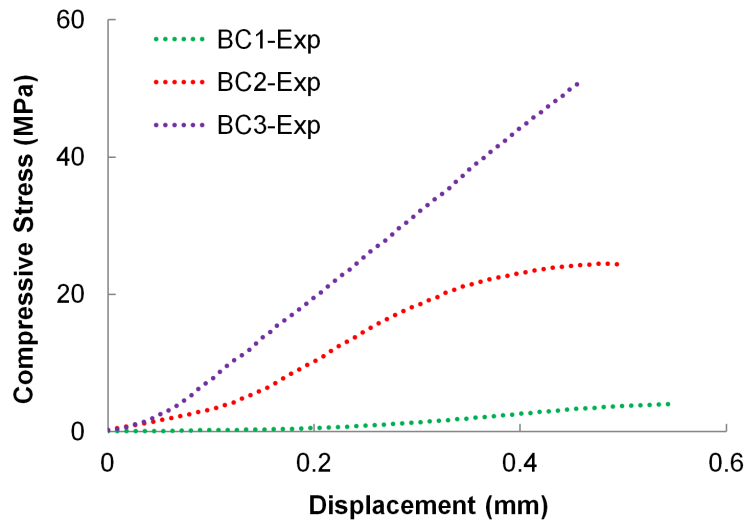

(a)

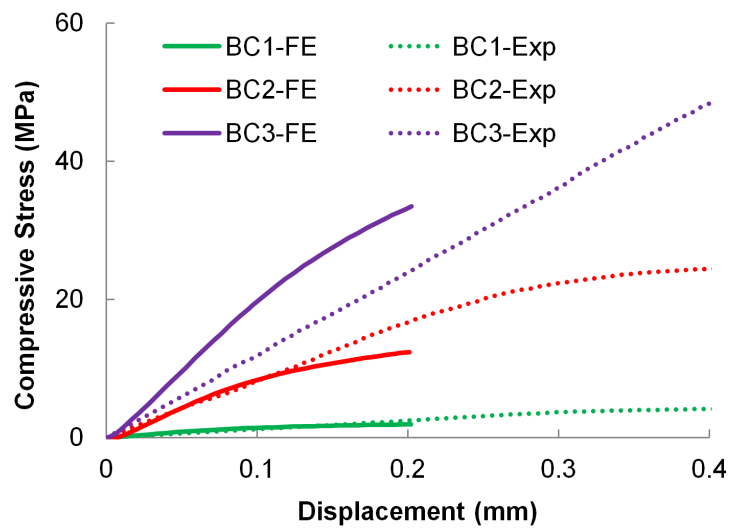

(b)

Fig.3 
(a)
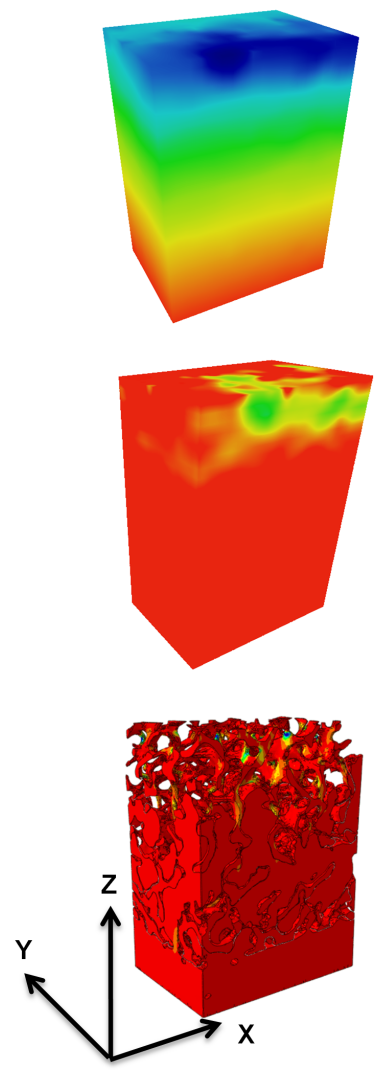

(a)

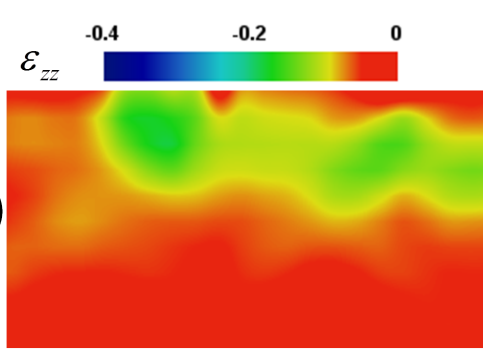

(b)

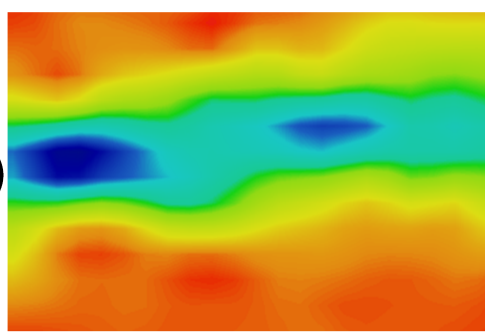

$(\mathrm{c})_{\uparrow}^{\mathrm{Z}}$ x (b)
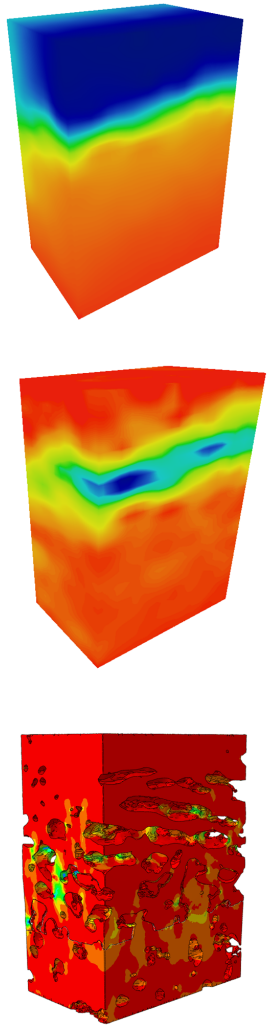

Fig.4

0\% Strain
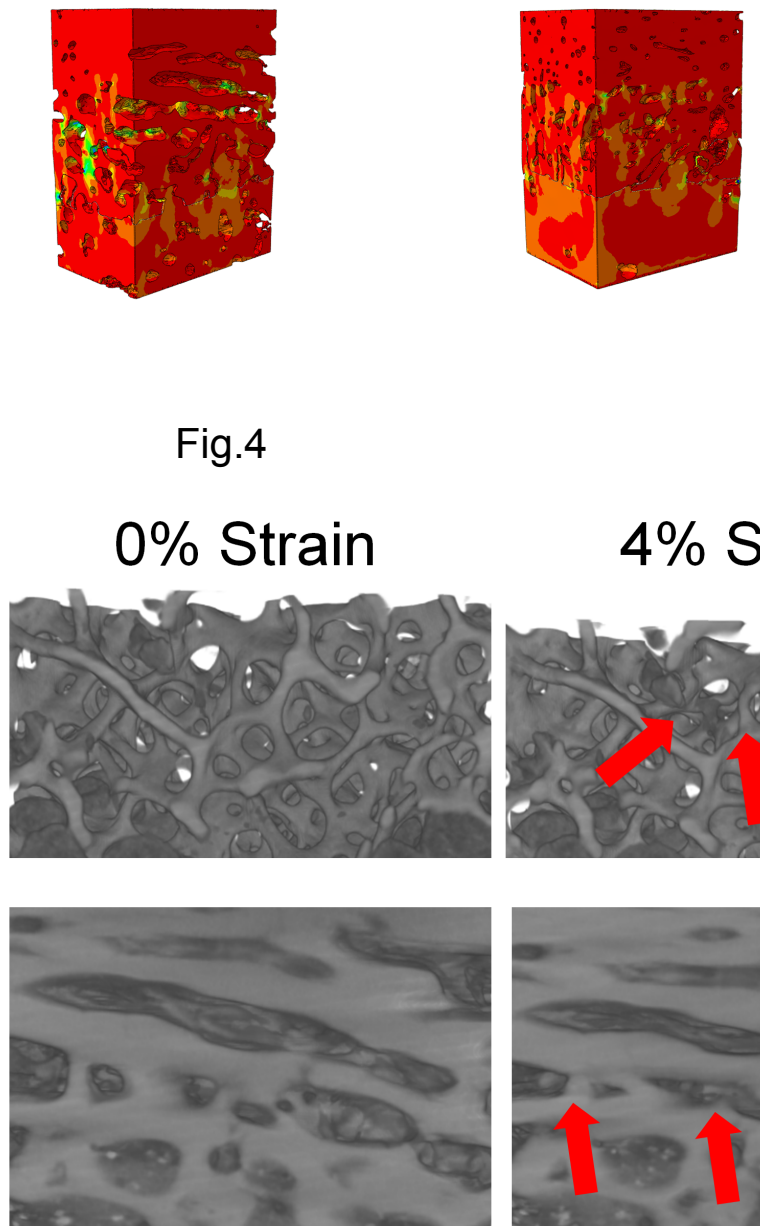
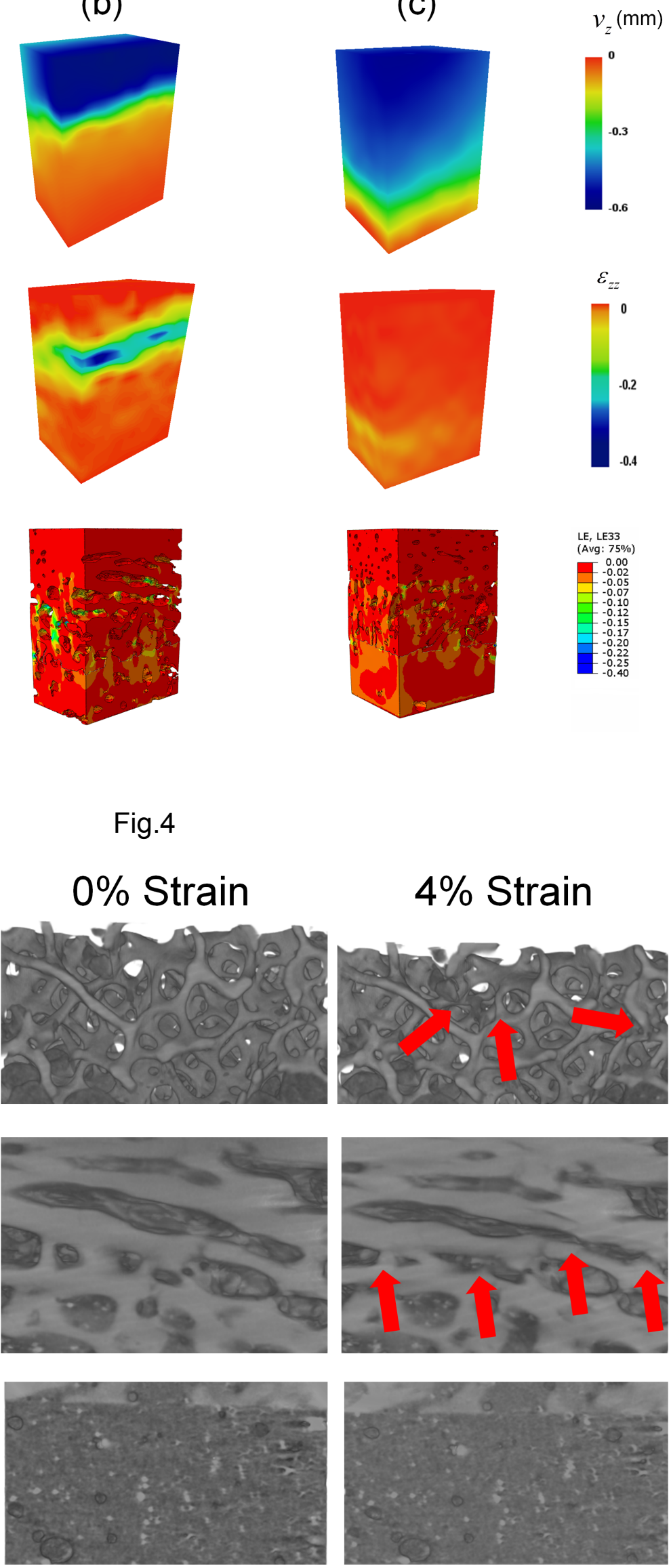
Fig.5

(a)

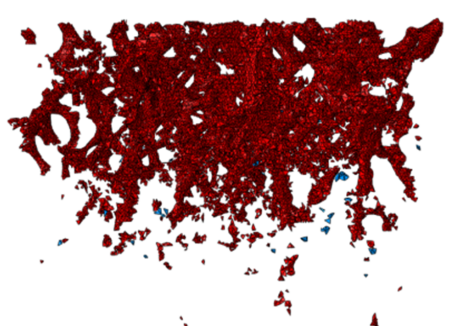

(b)

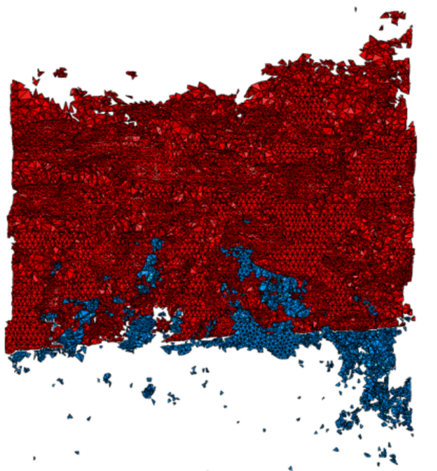

(c)

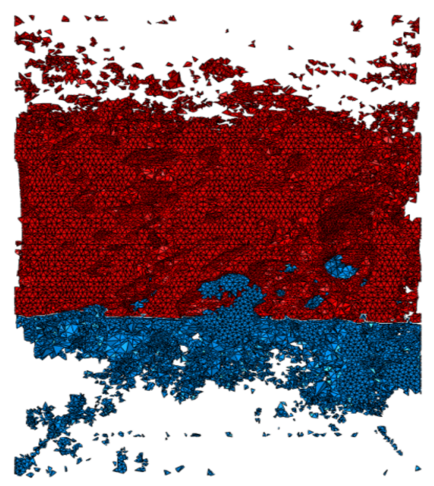

Fig.6 


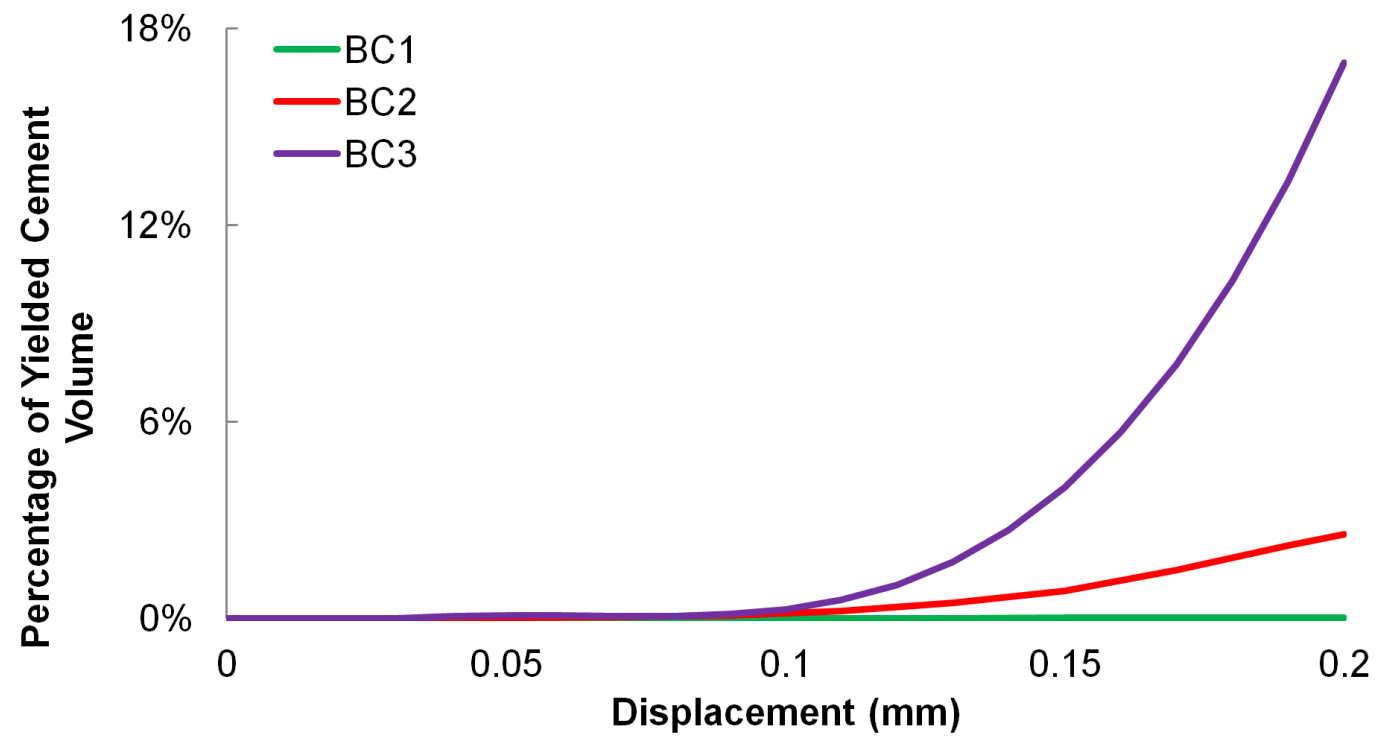

(a)

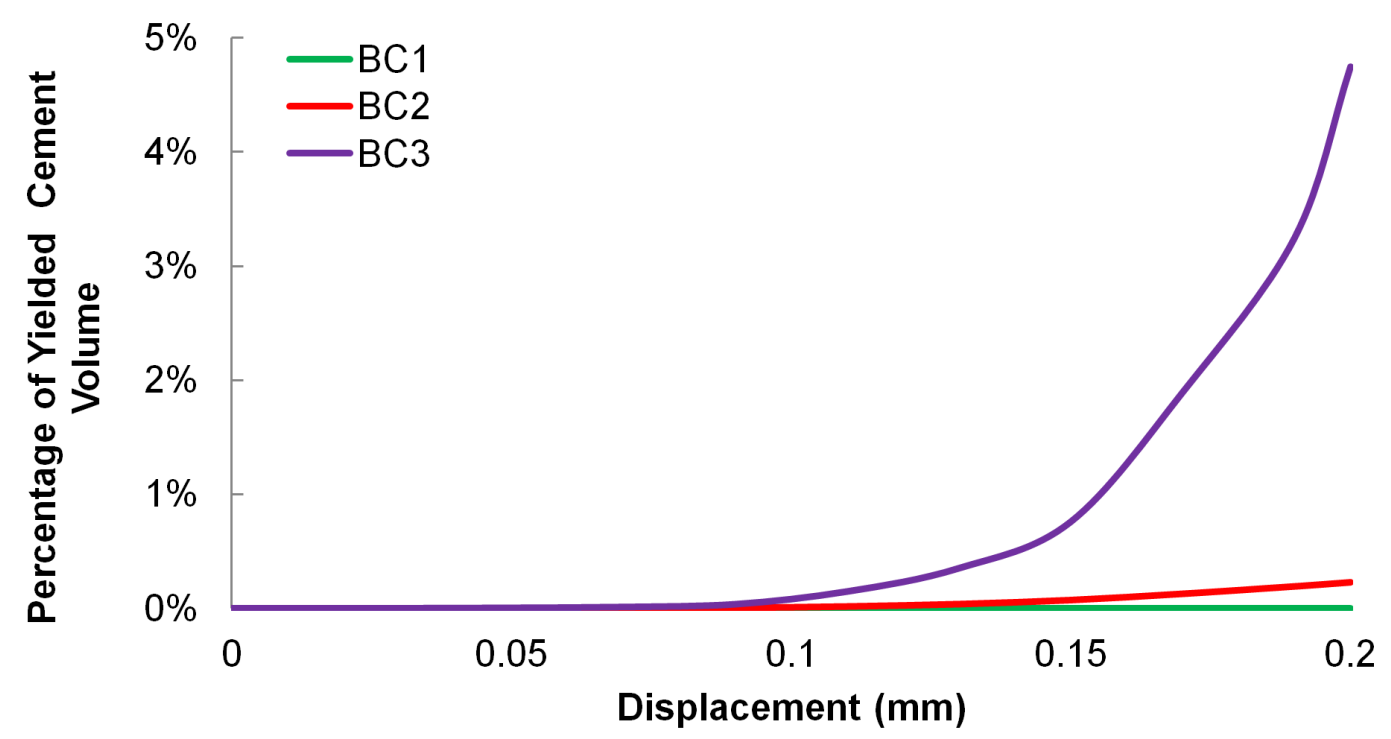

(b)

Fig.7 


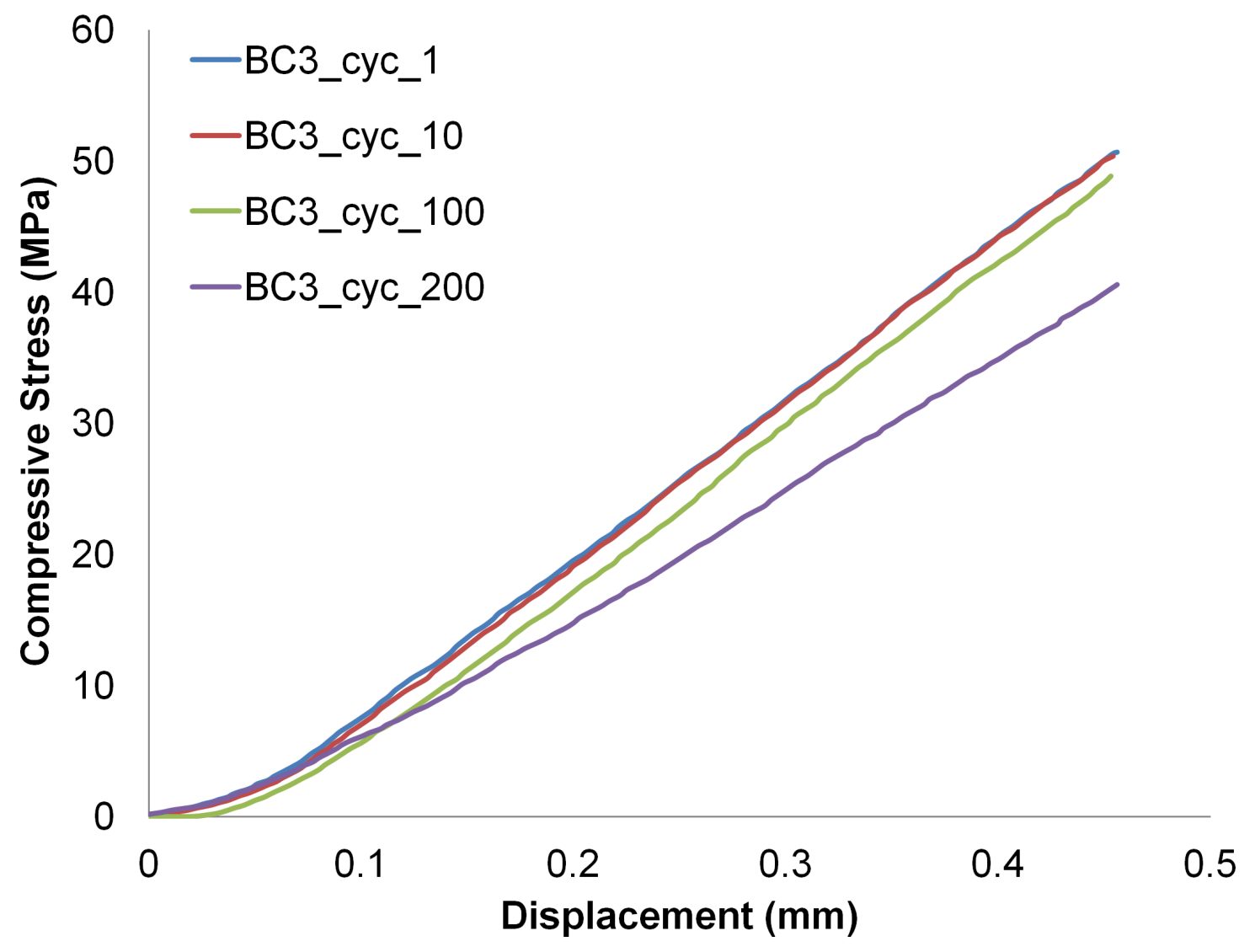

Fig.8 
(a)
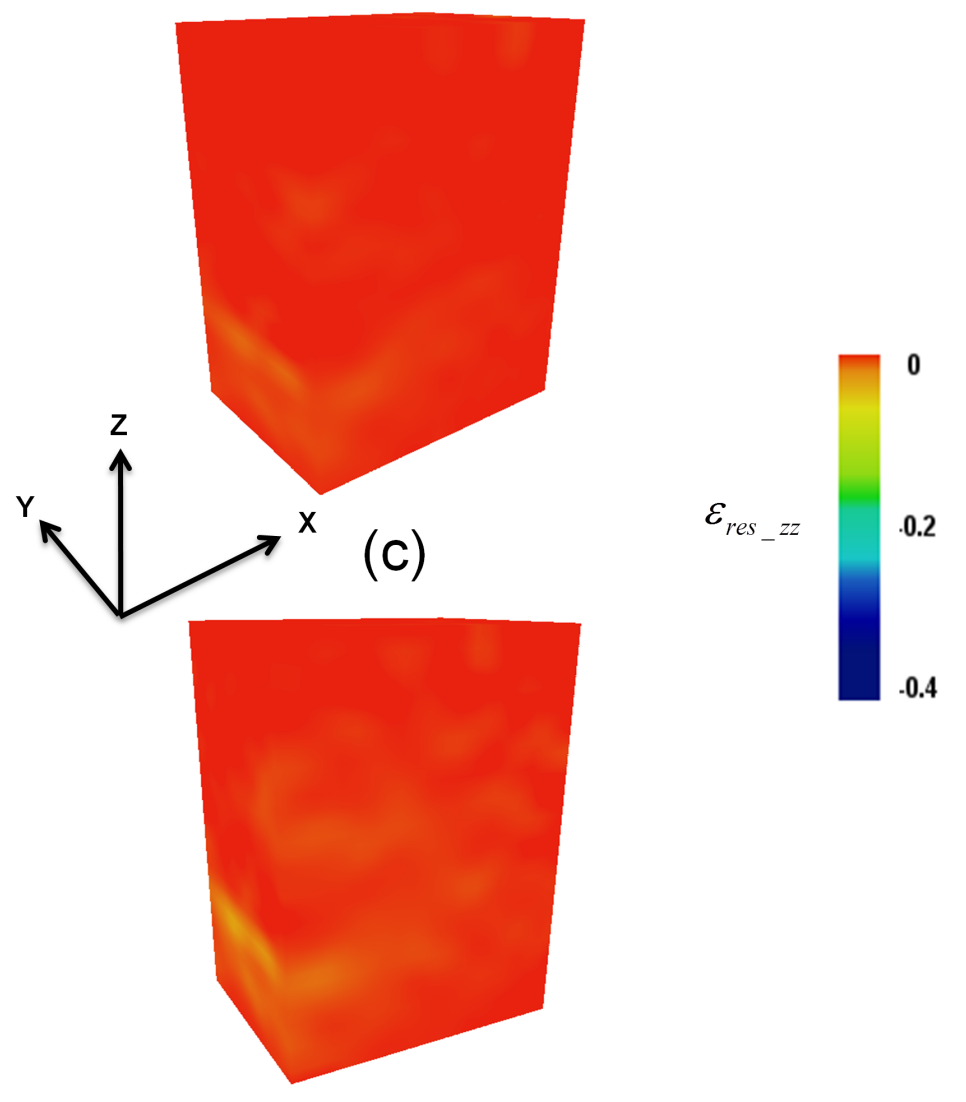

Fig.9

$\varepsilon_{\text {res_zz }}{ }^{-0.4}$

(a)

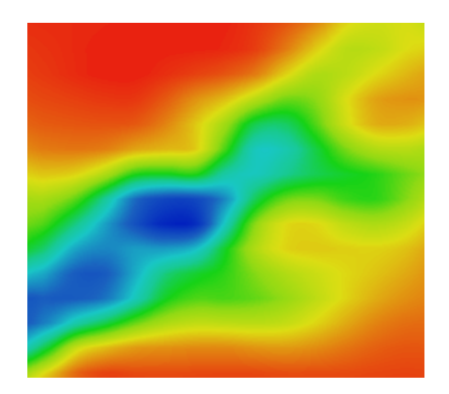

(b) $z$

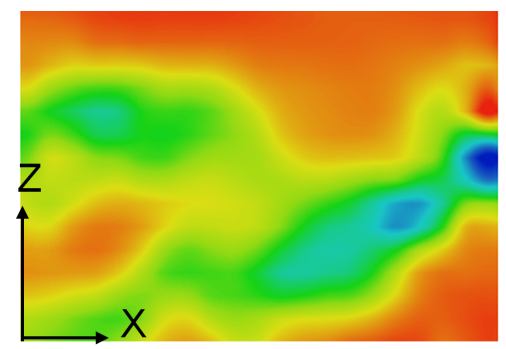

(b)

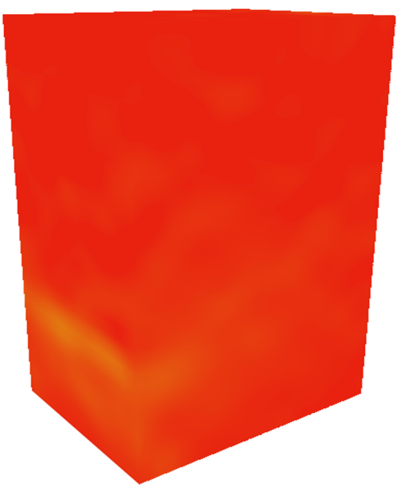

(d)

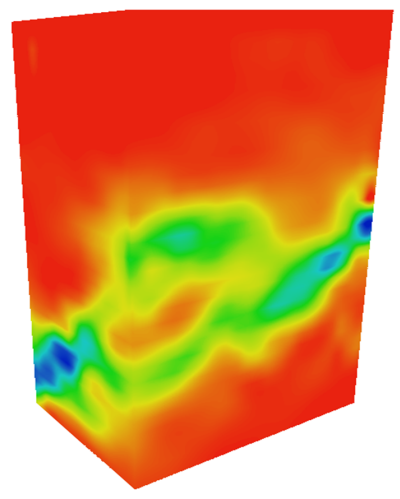

$0 \%$ Strain $(0$ cycles $) \quad 0 \%$ Strain (200 cycles)
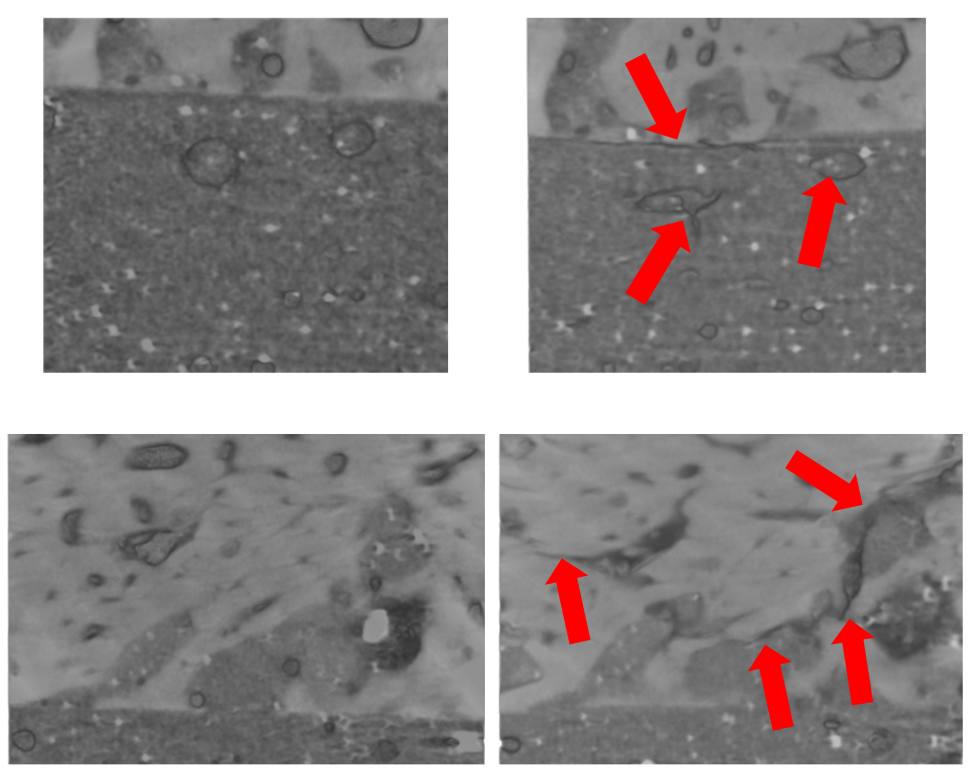
Fig.10 